\title{
Scale-invariance underlying the logistic equation and its social applications
}

\author{
A. Hernando ${ }^{1}$, A. Plastino ${ }^{2,3}$ \\ 1 Laboratoire Collisions, Agrégats, Réactivité, IRSAMC, \\ Université Paul Sabatier 118 Route de Narbonne 31062 - Toulouse CEDEX 09, France \\ ${ }^{2}$ National University La Plata, IFLP-CCT-CONICET, C.C. 727, 1900 La Plata, Argentina \\ 3 Universitat de les Illes Balears and IFISC-CSIC, 07122 Palma de Mallorca, Spain
}

\begin{abstract}
On the basis of dynamical principles we derive the Logistic Equation (LE), widely employed (among multiple applications) in the simulation of population growth, and demonstrate that scaleinvariance and a mean-value constraint are sufficient and necessary conditions for obtaining it. We also generalize the LE to multi-component systems and show that the above dynamical mechanisms underlie large number of scale-free processes. Examples are presented regarding city-populations, diffusion in complex networks, and popularity of technological products, all of them obeying the multi-component logistic equation in an either stochastic or deterministic way. So as to assess the predictability-power of our present formalism, we advance a prediction, regarding the next 60 months, for the number of users of the three main web browsers (Explorer, Firefox and Chrome) popularly referred as "Browser Wars".
\end{abstract}

\section{INTRODUCTION}

It is well-known that the logistic equation (LE) (sometimes called the Verhulst model or logistic growth curve) is a phenomenological model of population growth first published by Pierre Verhulst in the 1840's. The model is continuous in time, but a modification of the pertinent equation to a discrete quadratic recurrence equation known as the logistic map is also widely used. The continuous version of the logistic model for the evolution of the population $x(t)$ is described by the differential equation

$$
\dot{x}(t)=k x(t)\left(1-\frac{x(t)}{N}\right),
$$

where $k$ is the Malthusian parameter (rate of maximum population growth) and $N$ is the so-called carrying capacity (i.e., the maximum sustainable population). The LE has as a solution

$$
x(t)=\frac{N}{1+(N / x(0)-1) e^{-k t}},
$$

i.e., the sigmoid function. The discrete version of the LE is the celebrated logistic map. A typical application of the logistic equation refers to a 1838-model of population growth, originally due to Verhulst, in which the rate of reproduction is proportional to both the existing population and the amount of available resources, all else being equal. The Verhulst equation was published after he had read Thomas Malthus' An Essay on the Principle of Population. Verhulst derived his logistic equation to describe the self-limiting growth of a biological population. Today, proper referencing to the logistic equation's variegated applications to multiple fields of endeavor would require pages and pages of citations. Of this immense body we just mention, as a tiny sample ${ }^{1,7}$.

Some ad-hoc LE-deductions have been previously published in a case-by-case basis. One such demonstration is that provided by A. D. Zimm for companies or firms ${ }^{8}$, that grow according to their commercial successfulness with a classic linear Marshallian price-volume relationship. Other analytical derivations are also found with some ad-hoc assumptions 619 . Out present goal is to describe an universal and generic dynamical mechanism that leads in natural fashion to the logistic equation. The above cited derivations do not have a dynamical character, as ours does. Our procedure is based on

- scale symmetry and

- a mean-value constraint.

We will show that these are necessary an sufficient conditions for a LE-derivation. The two items above are empirically known to be related to the $L E^{6 / 8 \mid 9]}$ but they are used here for the first time as its pure mathematical basis.

\section{DERIVATION FROM DYNAMICAL PRINCIPLES}

Consider an $n$-components system, each of them characterized by a population $x_{i}$. Let us further assume that a multiplicative, time-evolution of population takes place via free proportional growth, i.e.,

$$
\dot{x}_{i}(t)=k_{i}(t) x_{i}(t),
$$

where $k_{i}$ is the growth-ratio per unit-time for the $i$-th component. Scale-symmetry is here apparent so that it proves convenient to transform coordinates to $u_{i}=$ $\log \left(x_{i}\right)$ as in Refs. 1213. Thereby one is led to the linear equation

$$
\dot{u}_{i}(t)=k_{i}(t),
$$

where the scale-invariance of $x$ is now a translational invariance in $u$. We assume that the total population is finite, namely

$$
\sum_{i=1}^{n} x_{i}(t)=\sum_{i=1}^{n} e^{u_{i}(t)}=N,
$$


and that also $n$ remains constant, so that $\langle x\rangle=N / n$. For each arbitrarily small time-interval $\Delta t$, the $u$-population grows freely via

$$
u_{i}^{\prime}(t+\Delta t)=u(t)+\Delta t k_{i}(t)
$$

but conservation of the mean value $\left\langle e^{u}\right\rangle$ demands a global "self-correction" process that should respect the original symmetry of the system (translational for $u$ ). Accordingly,

$$
u_{i}(t+\Delta t)=u_{i}^{\prime}(t+\Delta t)+A,
$$

where $A$ is a value that guarantees fulfillment of $\sum_{i=1}^{n} e^{u_{i}(t+\Delta t)}=N$. This is achieved if

$$
A=-\ln \left[\frac{1}{N} \sum_{j=1}^{n} e^{u_{j}^{\prime}(t+\Delta t)}\right]=-\frac{\Delta t}{N} \sum_{j=1}^{n} k_{j}(t) e^{u_{j}(t)},
$$

where a Taylor-expansion to first order is justified since $\Delta t$ is arbitrarily small. One is then led to

$$
u_{i}(t+\Delta t)=u_{i}(t)+\Delta t\left[k_{i}(t)-\frac{1}{N} \sum_{j=1}^{n} k_{j}(t) e^{u_{j}(t)}\right] .
$$

In the continuum-limit one finds

$$
\dot{u}_{i}(t)=k_{i}(t)-\frac{1}{N} \sum_{j=1}^{n} k_{j}(t) e^{u_{j}(t)}
$$

that written in $x$-parlance leads to what we call the multi-component logistic equation (MCLE)

$$
\dot{x}_{i}(t)=x_{i}(t)\left(k_{i}(t)-\frac{1}{N} \sum_{j=1}^{n} k_{j}(t) x_{j}(t)\right) .
$$

A matrix version of this equation is presented in the Appendix, together with with some applicability perspectives. It is easy to check that the MCLE retains the original scale-symmetry of $x$, and also exhibits translational symmetry in $k$. The latter allows for some arbitrariness in the definition of the $k_{i}$ rates in the fashion $k_{i}^{\prime}=k_{i}-k_{0}$. The same results obtain for primed or unprimed $k$ 's. If the $k_{i}$ are constant, or exhibit just a slow dependence on $t$ (quasi-statics), the solution to the MCLE is

$$
x_{i}(t)=\frac{N x_{i}(0) e^{k_{i} t}}{\sum_{j=1}^{n} x_{j}(0) e^{k_{j} t}},
$$

where $x_{i}(0)$ are the initial conditions of the evolutiveprocess. The logistic equation is directly derived from the MCLE Eq. (3) in a straightforward fashion. We recover Eq. (1) by i) considering a bi-component system $(n=2)$, ii) defining $x(t) \equiv x_{1}(t), k \equiv k_{1}-k_{2}$, and iii) taking into account that $x_{2}(t)=N-x_{1}(t)$. Similarly, the sigmoid function Eq. (2) is recovered from Eq. (4) with the same assumptions. The second component acts here as a population-reservoir and the first component becomes the only evolutive degree of freedom.

\section{POSSIBLE PHYSICAL REGIMES}

According to the nature of the growth-ratios $k_{i}$, variegated kinds of processes can be described by the MCLE.

1. Constant values or deterministic time-dependencies lead to mechanical systems exhibiting deterministic evolution while

2. adding noise to the pertinent mean values gives rise to stochastic systems, with interesting behaviors and applications.

Without aiming to be exhaustive, we consider here three different tableaus for MCLE-applicability, according to the amount of 'noise' in the system: totally stochastic (or thermodynamic regime), an intermediate level of randomness (involving diffusive processes), and totally deterministic dynamics.

\section{A. Thermodynamic regime}

Consider a multi-component case with dozens or hundreds of elements, and a very high level of noise. Assume that each $k_{i}$ describes the derivative of a Wiener process. We write $k_{i}(t)=\bar{k}_{i}+\sigma_{i} \xi(t)$, where $\bar{k}_{i}$ is the time-average of $k_{i}, \sigma_{i}$ the standard deviation measured in a certain interval $\Delta t$, and $\xi(t)$ an independent normal-distributed random number. Defining $\left\langle\sigma^{2}\right\rangle=\sum_{i=1}^{n} \sigma_{i}^{2} / n$, one asserts that we thermalize the system if i) $\left|\sigma_{i}-\sigma_{j}\right|^{2} /\left\langle\sigma^{2}\right\rangle \ll 1$ and ii) $\left|\bar{k}_{i}-\bar{k}_{j}\right|^{2} /\left(\left\langle\sigma^{2}\right\rangle \Delta t\right) \ll 1, \forall i, j$, i.e., if all elements exhibit similar deviations and the differences between mean values are much smaller than the noise. If $n$ is large enough (as stated above, in the hundreds), dynamical equilibrium is encountered after some finite time, meaning that the system is well-described by the MaxEnt approach. The MaxEnt solution for scale-free systems describes an equilibrium density $p_{X}(x)$ that follows the general form 12

$$
p_{X}(x) d x=\exp \left[-\sum_{a} f_{a}(x)\right] \frac{d x}{x},
$$

where $f_{a}(x)$ is the $a$-th constraint of the system. For a constraint in the normalization ( $n$ is invariant) we have $f_{\langle 1\rangle}(x)=\mu$, and for one in the mean value we write $f_{\langle x\rangle}(x)=\lambda x$, where $\mu$ and $\lambda$ are constants, univocally determined by the fulfillment of each constraint. The density-distribution obeys in this case the relation 13

$$
p_{X}(x) d x=\frac{1}{\Gamma\left(0, \lambda x_{0}\right)} \frac{e^{-\lambda x}}{x} d x ; \quad x_{0} \leq x<\infty,
$$

where $\Gamma$ is the incomplete gamma function and $x_{0}$ is the smallest allowed population for the elements (that can be 1). The rank-distribution is then written as

$$
x=\frac{1}{\lambda} \Gamma^{-1}\left[0, \Gamma\left(0, \lambda x_{0}\right) r / n\right],
$$


where $r$ is the (continuous) rank from 0 to $n$, and $\Gamma^{-1}(z)$ denotes the inverse function of $\Gamma: \Gamma\left(\Gamma^{-1}(z)\right)=z$. The value of $\lambda$ is obtained from the mean value

$$
\frac{e^{-\lambda x_{0}}}{\lambda \Gamma\left(0, \lambda x_{0}\right)}=\frac{N}{n} \text {. }
$$

In order to test the above relationships we have carried out a calculation that simulates human population dynamics. We also compare the result with empirical data regarding city and place-populations, using as example data from Ohio State (United States) $\underline{14}$. We consider $n=1000$ random walkers that mimic the population of cities, and set the total population to $N=6000000$. We fix the minimum allowed size at the Dunbar's number $\underline{13}$ $x_{0}=150$ people (empirically known to be the usual size of small human communities, and related to the maximum of social relationships/links that a human can comfortably handle). We make the walkers to stochastically obey the MCLE Eq. (3) so as to simulate migration patters between cities, with a constant total population. We evolve the walkers in small intervals $\Delta t=0.03$ and generate gaussian-distributed random numbers at each interval for each $k_{i}$, using $\sigma_{i}=1$ for all $i$. Due to the translational symmetry in $k$, we can set $\bar{k}_{i}=0$ for all $i$. To respect the minimum size, a walker' 'move' is not accepted if it leads to a value lower than the Dunbar's one. We start all walkers at $x_{i}=N / n$. After some iterations we get the equilibrium distribution of Fig. 1, which perfectly fits the MaxEnt prediction $\left(\lambda=0.00533\right.$ humans $\left.^{-1}\right)$. The available empirical data covers years 2010, 2000 and 1990 with $n=1204,1015$, and 928 cities and places, respectively. We discard places with populations of under 150 people (67, 48 and 39 centers respectively). We also discard very large cities (their potential economic correlation with the rest of the country compromises the hypothesis of isolated systems). Excluding the four largest cities, the total population is $N=6318170,6019960$, and 5477830, respectively. We have checked the proportional growth condition comparing $\log \left(x_{i}\right)$ vs. $\left|\dot{x}_{i} / x_{i}\right|^{2}$ (or equivalently, $u_{i}$ vs. $\left|\dot{u}_{i}\right|^{2}$ ). No correlation between these two observables is expected for scale-invariance. We have found a correlation coefficient of 0.0018 , as shown in Fig. 1, thus confirming the proportional growth hypothesis (the same correlation coefficient in the precedent simulation is 0.0027). According to Eq. (6), the predicted values of $\lambda$ are $0.00585,0.00507$, and 0.00513 , respectively. A direct fit of the data to the form Eq. (5) yields $\lambda=0.00636(2)$, $0.00502(3)$, and $0.00522(3)$, respectively, close enough to the former values so as to confirm the MaxEnt prediction.

\section{B. Intermediate regime}

Let us pass now to consideration of an intermediate stochastic regime in the bi-component case. One party acts as a population-reservoir while the other obeys Eq. (1). Defining the new variable

$$
y(t)=-\log (N / x(t)-1),
$$

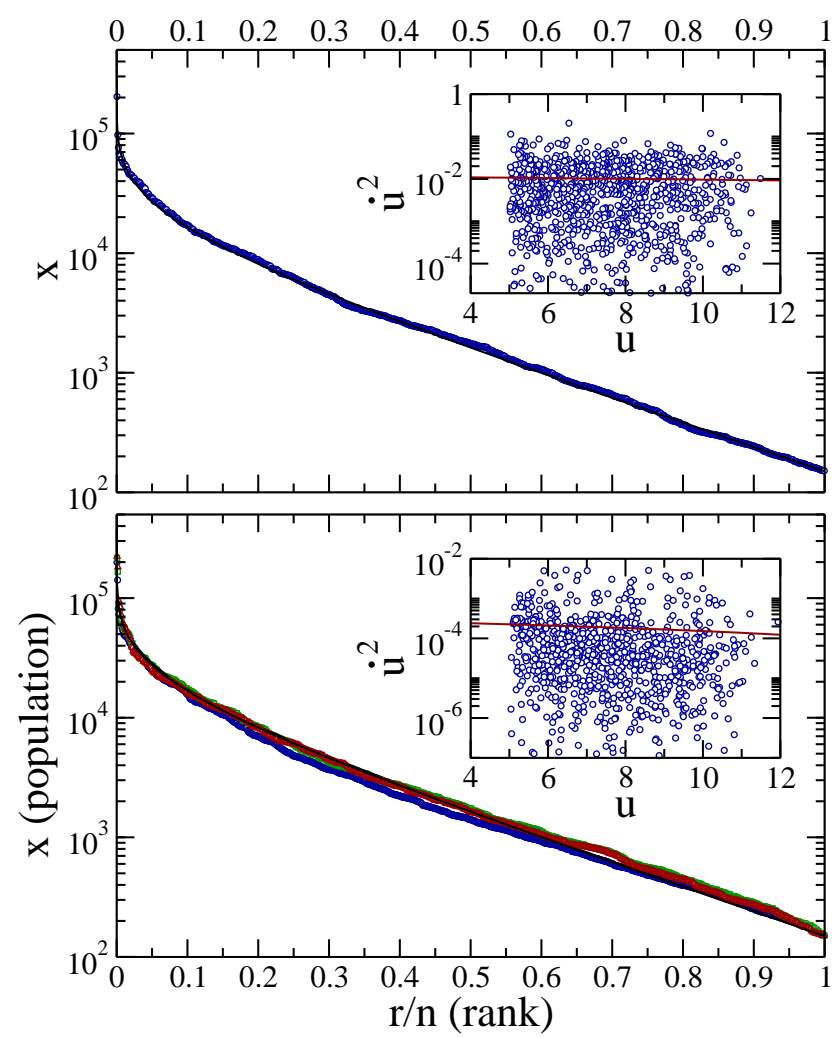

Figure 1: Top panel: behavior of random walkers obeying the stochastic MCLE (blue dots) compared with the associated MaxEnt prediction (black line). Inset: walkers' squaredrelative-growth $\left|\dot{u}_{i}\right|^{2}$ vs. logarithmic size $u$. There is no correlation in view of the linear regression (red line). Bottom panel: Rank-distributions for Ohio (red: year 2010; green: year 2000; blue: year 1990) and rank-distribution of random walkers obeying the stochastic MCLE (yellow) compared with the corresponding MaxEnt predictions (black lines). Inset: same as upper panel with the empirical data. In view of the regression line, no correlation is detected.

the LE linearizes itself for $\dot{y}(t)=k$. The rate $k$ is here again the derivative of a Wiener process $k(t)=\bar{k}+\sigma \xi$, but we now include a drift obeying $|\bar{k}|^{2}>\sigma^{2} \Delta t$. Working with an ensemble of independent walkers following this equation is equivalent to handling Brownian motion in $y$-space. Consequently, the usual diffusion equation for the density of walkers for $p_{Y}(y, t)$ ensues ${ }^{15}$

$$
\partial_{t} p_{Y}(y, t)=-\bar{k} \partial_{y} p_{Y}(y, t)+D \partial_{y}^{2} p_{Y}(y, t),
$$

where $D$ is the diffusion-coefficient, related to $\sigma_{k}$ via $\sigma_{k}=$ $\sqrt{2 D / \Delta t}$, and $\Delta t$ stands for the time-interval used in the random-walk numerical simulation. The diffusionequation's kernel is a Gaussian

$$
p_{Y}(y, t) d y=\frac{1}{\sqrt{4 \pi D t}} e^{-\frac{\left(y-y_{0}-\bar{k} t\right)^{2}}{4 D t}} d y,
$$

with $y_{0}$ a reference-value. If at $t=0$ all walkers are located in $x$-space at $x_{0}=N /\left(1+e^{-y_{0}}\right)$, they will later 
evolve via

$$
p_{X}(x, t) d x=\frac{1}{\sqrt{4 \pi D t} x(1-x / N)} e^{-\frac{\left(\log (N / x-1)-y_{0}+\bar{k} t\right)^{2}}{4 D t}} d x,
$$

since $p_{X}(x, t) d x=p_{Y}(y, t) d y$.

We have verified this prediction with a diffusion process taking place inside a scale-free ideal network (SFIN) $)^{16}$, a random network with a degree-distribution following the scale-free ideal gas one $p(c) \sim c^{-1}$, where $c \leq c_{M}$. We have generated a SFIN of $N=20000$ nodes with a maximum degree of $c_{M}=100$-connections and carried out a multitude of cluster-growth processes $16 / 17$. Diffusion in networks generally starts i) by using a randomly chosen node as a seed, ii) with its first neighbors being added to the cluster in the first iteration, iii) and the neighbors of those "first" neighbors, afterwards (and so on). The process ends when all nodes of the network belong to the cluster. The size of the cluster at each iteration depends on the particular node selected as seed, via its position inside the network. We depict in Fig. 2 the result of a large number of these processes, indicating the size of the cluster at each iteration. All of them start with $x=1$ and end up with $x=N$, but processes exhibit deviations at intermediate steps. The associated median clearly follows a logistic evolution, as shown in Fig. 2. Changing to the variable $y$ of Eq. (7) we find a straight line with slope $\bar{k}=3.09$. The deviations can be described by $y$ random walkers with $\sigma=0.7(\Delta t=1)$. The statistics of the processes can be nicely described with Eq. (8) via $x_{0}=1, D=0.245$, and with the above value of $k$, as illustrated by the comparison depicted in Fig. 2.

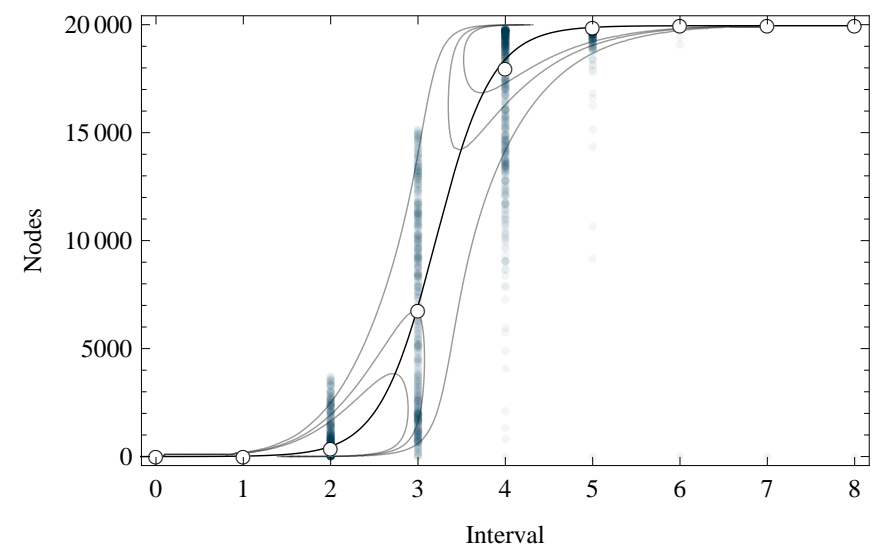

Figure 2: Diffusion inside a scale-free ideal network (dots) compared with the analytical one provided by Eq. (8), derived from the logistic equation.

\section{Deterministic regime}

We study now the deterministic evolution of a multicomponent system with few elements and very low level of (or without) noise. Assume that the growth-ratios $k_{i}$ are now constants or represent a quasi-static evolution in time. Assume further that we have data about the temporal population-evolution but do not known the explicit values (or the tendency) of the $k_{i}$ rates. These can be easily obtained from the solution of MCLE Eq. (3) taking advantage of its property of translational symmetry in $k$. By arbitrarily setting $k_{1}=0$, all the remaining values are obtained from the population data thanks to the functions

$$
h_{i}(t)=\log \left[\frac{x_{i}(0)}{x_{i}(t)} \frac{x_{1}(t)}{x_{1}(0)}\right] .
$$

If the growth-ratios are constants, $h_{i}(t)=k_{i} t$, the entire evolution-path can be predicted (for any arbitrary time). If our functions $h_{i}(t)$ exhibit small time-fluctuations we can parameterize them, via a fitting procedure, to any given analytical form. The desired solution is obtained by substituting the arguments in the exponentials of Eq. (3) by these functions $k_{i} t \rightarrow h_{i}(t)$. We have tested this last statement using data regarding web-browsers' statistics so as to study the past and future of the (popularly called) Browser Wars 18 . We considered the $n=3$ system composed of Microsoft Explorer (E), Mozilla Firefox (F), and Google Chrome (C). Our analysis of the popularity of each uses data from w3schools $\sqrt{19}$ and statcounter 20 (depicted in Fig. 3).

We take $N=100 \%$ and choose the origin $t=0$ at March 2012 (as this communication was being written). Setting $k_{E}=0$ we have applied Eq. (9) to the data, finding a small dependence on time in both $k_{F}$ and $k_{C}$. We show in Fig. 3 that the functions $h_{i}(t)$ can be nicely fitted to a simple exponential form $h(t)=a e^{-b t} t+c$ (that can be regarded as a pure exponential time-dependence of $k$ plus a correction on the initial value $x(0)$ via $e^{c}$ ). Note that the small fluctuations of the data become more apparent as we approach the reference point at $t=0$. However, our accuracy remains sufficiently high for our purposes. We obtain

$$
\begin{aligned}
& h_{F}(t)=0.0074(5) \exp [-0.021(1) t] t-0.008(14) \text { and } \\
& h_{C}(t)=0.0579(24) \exp [-0.0097(10) t] t+0.104(26),
\end{aligned}
$$

for w3schools, and

$$
\begin{aligned}
& h_{F}(t)=0.0022(5) \exp [-0.043(6) t] t+0.026(13) \text { and } \\
& h_{C}(t)=0.055(2) \exp [-0.015(1) t] t+0.015(27),
\end{aligned}
$$

for statcounter, that are compared in the top panels of Fig. 3 to empirical data. Our predictions for the popularity evolution are evaluated using Eq. (4) and substituting $k_{E} t, k_{F} t$, and $k_{C} t$ by the above described extrapolations of $h_{E}, h_{F}$, and $h_{C}$. A proper correction is finally added in the later case to improve the fitting by using $N^{\prime}=1.03 N$. We depict in Fig 3 our monthly prediction for the next 5 years regarding browsers' usage. In the two reported instances, Google Chrome grows till coming ahead in the competition, saturating effects being noticeable at $80 \%$ and $60 \%$, respectively. Thus, according to our prediction, Google Chrome will win the competition but it will 
not acquire such a dominant position as the MS Explorer attained in the past.

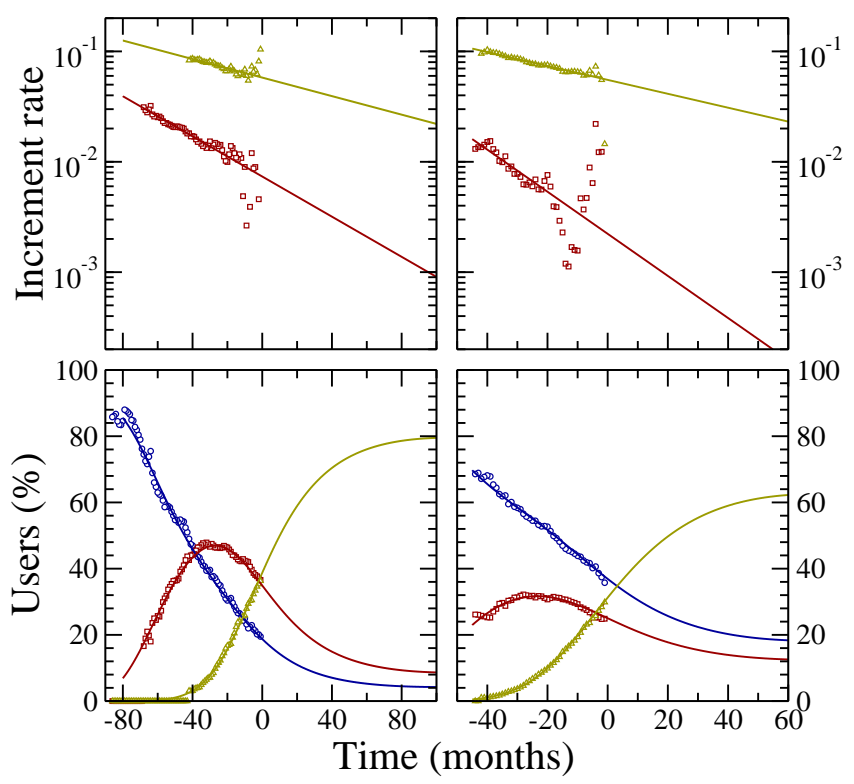

Figure 3: Left panels: data from w3schools. Right panels: data from statcounter. Top panels: increment rate of M Firefox (red squares) and G Chrome (yellow triangles) defined as $(h(t)-c) / t$ relative to MS Explorer (see text), compared with the analytical fit (solid lines). Bottom panels: relative users of MS Explorer (blue circles), M Firefox (red squares) and G Chrome (yellow triangles), compared with our prediction (lines).

\section{CONCLUSIONS}

We have been able here to demonstrated that scaleinvariance and a mean-value constraint are sufficient and necessary conditions for obtaining the LE from first dynamical principles. Then, the LE was generalized to multi-component systems. This allowed us to show that these dynamical mechanisms underlie interesting scalefree processes, which was illustrated with reference to city-populations phenomena, diffusion in complex networks, and popularity of Net Browsers.
Appendix A: Generalization of the multi-component logistic equation to a matrix equation

We generalize here the formalism discussed above. If we define a new set of variable $\chi_{i}=\sqrt{x_{i} / N}$, the total-population's constraint can be recast in the fashion $\sum_{i=1}^{n} \chi_{i}^{2}=1$. This condition becomes formally equivalent to the conservation of the modulus of a vector $\chi=\left\{\chi_{i}\right\}_{i=1}^{n}$ in a $n$-dimensional space. We can also generalize the definition of the growth-ratios $k_{i}$, promoting them to a matrix $K_{i j}=k_{i} \delta_{i j}$, and write the MCLE as a matrix equation. Using bra-ket notation $\chi=|\chi\rangle$ one has

$$
\partial_{t}|\chi\rangle=\frac{1}{2}\left\{K-\frac{\langle\chi|K| \chi\rangle}{\langle\chi \mid \chi\rangle}\right\}|\chi\rangle
$$

This equation is formally identical to that used in quantum physics to find the ground state wave-function of a Hamiltonian (here, $K$ ). We are speaking of the Imaginary Time Method (ITM) widely used in the literature 21 . The mean-value term is also used to guarantee the conservation of the normalization of $|\chi\rangle$ during the process. All our examples can be regarded as particular applications of this formalism, calling attention to the "functional" definition of our effective 'Hamiltonians' $K$. In our examples $K$ has a diagonal form, which only indicates that we were working in the eigenbasis of the dynamics defined by $K$. A generalized definition can include off-diagonal terms as well, indicative of some kind of interaction between populations. Density functional theories (DFT) also use the above equation for manybody quantum systems 22 . A phenomenological Hamiltonian is defined by means of a parametric functional form, than can also be a functional of the own state-vector $\chi$. The associated parameters are chosen so as to reproduce well-known empirical facts regarding the system of interest. We expect that the bridge we have here built up between the MCLE and the DFT can open new vistas with respect to the possibility of studying scale-free systems. Such approach would take advantage of the huge experience accumulated regarding DFT methods.
1 S. Jannedy, R. Bod, J. Hay, Probabilistic Linguistics (MIT Press, Cambridge, Massachusetts, 2003).

2 N. A. Gershenfeld, The Nature of Mathematical Modeling (Cambridge University Press, Cambridge, UK, 1999).

3 S. E. Kingsland, Modeling nature: episodes in the history of population ecology (University of Chicago Press, Chicago, 1995).

${ }^{4}$ E. W. Weisstein, Logistic Equation, from Wolfram Research Mathworld, Repository hosted at UIUC.
5 M. Fuentes, H. Larrondo, M. T. Martin, A. Plastino, O. Rosso, Phys. Rev. Lett. 99 (2007) 154102.

${ }^{6}$ E. O. Wilson, W. H. Bossert. A Primer of Population Biology (Sinauer Associates, Sunderland, MA 01375, 1971).

7 J.P. Gabriel, F. Saucy, L. F. Bersier, Ecological Modelling 185 (2005) 147.

8 A. D. Zimm, Comp. \& Math. Org. Theo., 11 (2005) 37.

9 T. Royama, Analytical Population Dynamics (Chapman and Hall, London, 1992). 
10 E. T. Jaynes, (1957). Phys. Rev. 106, 620 (1957); 108, 171 (1957); IEEE Trans. Syst. Sci. \& Cyb. 4, 227 (1968).

11 A. Katz, Principles of statistical mechanics: the information theory approach (W. H. Freeman, San Francisco, 1967).

12 A. Hernando, A. Plastino, Variational Principle underlying Scale Invariant Social Systems. Pre-print (2012).

13 A. Hernando, A.R. Plastino, A. Plastino, Eur. Phys. J. B., accepted for publication (2012).

${ }^{14}$ Census bureau website, Government of USA, www.census.gov.

15 B. H. Lavenda, Nonequilibrium Statistical Thermodynamics (John Wiley \& Sons Inc., 1985).
16 A. Hernando, D. Villuendas, C. Vesperinas, M. Abad, A. Plastino, Eur. Phys. J. B 76, 87 (2010).

17 C. Castellano, S. Fortunato, and V. Loreto, Rev. Mod. Phys., 81, 591 (2009).

18 Wikipedia, Browser wars. http://en.wikipedia.org/wiki/Browser wars

19 http://www.w3schools.com/browsers/browsers stats.asp

20 http://gs.statcounter.com/

21 V. S. Popov, Phys. of Atom. Nuclei, 68 (2008) 686-708.

22 Parr, R. G.; Yang, W.Density-Functional Theory of Atoms and Molecules (Oxford University Press, New York. 1989) 